\title{
Direct Bonding SOI Wafer Based MEMS Cantilever Resonator for Trace Gas Sensor Applicaiton
}

\author{
Dong Ying, Gao Wei, You Zheng \\ Department of Precision Instruments and Mechanology, Tsinghua University, China
}

\begin{abstract}
A thermal driving and piezoresistive sensing MEMS cantilever resonator has been proposed and developed to construct trace gas detection sensors. The problem of integrating vibration structure, transducers and electric elements is the main concern in the design and fabrication of the resonator. In this paper, the parameters and the configuration of the resonator are discussed, the fabrication process and the test results are presented. Finite Element Analysis (FEA) has been carried out to optimize the configuration of the resonator to obtain high sensitivity and efficiency with a uniform temperature distribution that is propitious to the function of the gas sensing material. The fabrication process is based on direct bonding silicon-oninsulator (SOI) wafer and inductive coupled plasma (ICP) etching technology, which conciliate the semiconductor processes and the micromaching processes, and provide precise control of the resonator parameters. The experimental test results of the fabricated resonator agreed well with the calculation and simulation results and demonstrated that the proposed resonator was qualified to construct trace gas detection sensors.
\end{abstract}

Keywords —cantilever, gas sensor, resonator, SOI wafer

\section{INTRODUCTION}

Micromachined cantilevers have been proposed for a variety of sensor applications [1,2]. With a suitable driving mechanism cantilever can operate in resonant vibrating mode where causes of the resonant frequency shift can be detected with a frequency tracking mechanism. Several combinations of vibration driving and frequency tracking mechanisms can be applied to the micromachined cantilevers [3, 4]. For portable sensor application, it is necessary to integrate the driving and tracking transducers into the cantilever to form a MEMS resonator. Micromachined monocrystalline silicon cantilevers have inherently high quality factor (Q), providing the resonator with high frequency resolution. It has been demonstrated that micromachined resonant cantilever sensors can achieve nanogram-level to picogram-lever mass sensitivity, which attracts intensive interests for bio-chemical applications [5]. However, fabricating cantilever structure, transducers and electric paths and pads from a single crystal wafer involves complicated processes. Efforts have to be made to compromise the feasibility of the processes and the performances of the device [6]. We have developed a MEMS cantilever resonator for on-site trace gas detection application. Thermal driving and piezoresistive sensing combination was used. A fabrication process based on direct bonding SOI wafer was proposed to solve conflicts in different processes and to improve control precision of the device fabrication. In this paper the design, fabrication and test results of the MEMS cantilever resonator are presented.

\section{RESONATOR CONFIGURATION}

\section{A. Cantilever Dimension}

The operation principle of the resonant gas sensor is based on the following model [4]:

$$
\frac{\delta f}{f}=-\frac{1}{2} \frac{\Delta m}{m}
$$

where $m$ and $f$ are the mass and resonant frequency of the cantilever respectively, $\Delta m$ is the additional mass loading on the cantilever that is caused by the specific gas molecules being trapped in the sensing layer attached on the surface of the cantilever, and $\delta f$ is the changes of $f$ due to the mass loading. Therefore, through monitoring the frequency change $\delta f$, mass loading $\Delta m$ can be measured, and accordingly information about the gas concentration can be obtained.

It can be seen from the model that higher resonant frequency and less mass of the cantilever resonator provide better sensitivity for the sensor. Therefore the design principle of the cantilever dimension is that under the precondition of providing enough space to arrange the vibration driving and sensing elements on the cantilever surface, to get higher frequency and less mass. Taking the fabrication factors into consideration, the dimension of the cantilever with rectangle cross section is $1000 \mu \mathrm{m}$ long, $400 \mu \mathrm{m}$ wide and $10 \mu \mathrm{m}$ thick. The density of silicon is $2.23 \times 10^{-15} \mathrm{~kg} / \mu^{3}$, then the mass of the cantilever is about $9 \mu \mathrm{g}$. Calculation using classical formula [4] shows that the fundamental frequency of the cantilever is about $14 \mathrm{kHz}$. With a frequency resolution of $1 \mathrm{~Hz}$ which can be easily obtained by an ordinary counter, a mass resolution of $1 \sim 2 \mathrm{ng}$ can be obtained according to Eq. (1). Therefore trace gas detection can be expected with this cantilever dimension.

\section{B. Transducers Arrangement}

Thermal driving and piezoresistive sensing mode is adopted by the resonator. The heating resistors and the piezoresistors are integrated with the cantilever to construct a MEMS resonator. After the resonator is fabricated and packaged into a chip, the gas sensing material will be deposited onto the cantilever to construct a gas sensor. The position of heating resistors, piezoresistors and gas sensing layer on the surface of the cantilever affects the performance of the resonator and the sensor indeed. Trade-offs must be made in the consideration of the transducers arrangement. 
It is well known that stress increases to the most at the root of deflected cantilever, therefore to obtain the maximal signal to noise ratio the piezoresistors should be positioned in the area close to the root. Another common knowledge is that the highest efficiency can be obtained if the cantilever is driven at its free end. Also it has been understood that the resonant frequency has the most sensitivity to the mass loading occurring at the tip of the cantilever [7]. Meanwhile the temperature elevation and distribution on the cantilever due to heating has impact on the performance of the gas sensing material. Generally to say, proper temperature with a uniform distribution is propitious to the function of the gas sensing material.

FEA simulation has been carried out in order to optimize the transducers arrangement. The option we made is, two identic slits divide the cantilever into three areas and from the tip to the root of the cantilever there are gas sensing layer, heating resistors and piezoresistors ordinally in each of the three areas. The slits obstruct the heat conduction and produce uniform temperature elevation in the gas sensing area.

\section{Heating Resistors}

The thermal driving element is composed of four shuntwound resistor strips stretching along the width of the cantilever, as sketched in Fig. 1 where dashed lines indicate electrical connection. Using shunt-wound resistors instead of a single resistor can avoid local heating due to the nonuniform doping of the resistor strip. Each of the strips is $100 \mu$ long and $25 \mu \mathrm{m}$ wide and has $15 \mu \mathrm{m}$ space in between. Given the sheet resistance of the doping process $80 \sim 100 \Omega / \square$, the overall resistance of the thermal driving element is about $80 \sim 100 \Omega$. Through electrical-thermal-mechanical couple simulation by ANSYS, near $1 \mu \mathrm{m}$ deflection at the tip of the cantilever can be produced under the IC standard voltage $5 \mathrm{~V}$.

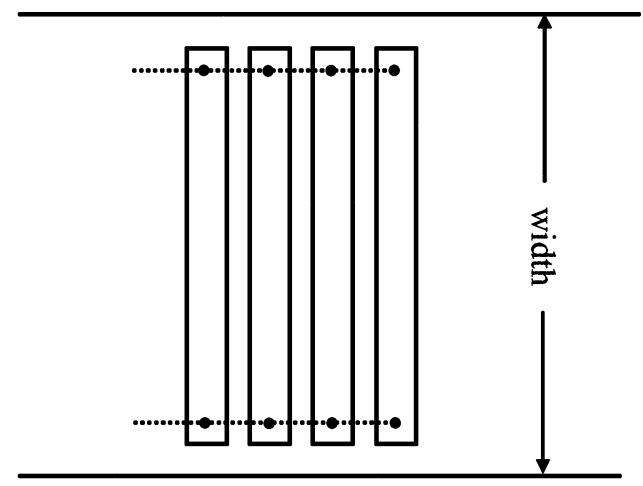

Figure 1. Sketch of the thermal driving element

\section{Piezoresistors}

The piezoresistors to construct the Wheatstone bridge are usually $1 \sim 5 \mathrm{k} \Omega$ for pressure or stress measurement applications. The resistor strips will be too long to be positioned within the cantilever if the same doping process as the heating resistors is used to fabricate the piezoresistors. Therefore the resistor strips must be made into zigzag shape just like those in pressure sensors. For the convenience of the connection and the compactness of the arrangement, three parallel resistor strips are connected in serial. Each of the strips is $150 \mu \mathrm{m}$ long and $15 \mu \mathrm{m}$ wide and has $15 \mu \mathrm{m}$ space in between as sketched in Fig.2. Because the connection path are vertical to the strips, reverse piezoresistive effect will be arosed. To eliminate the reverse effect, the resistance of the connection paths must be much smaller than the strips, which means they must be heavy doped. With this strategy the overall resistance of a piezoresistor is approximately three times the resistance of the resistor strip which is about $800 \sim 1000 \Omega$ if the same doping process as the heating resistors is used, and therefore $2.4 \sim 3 \mathrm{k} \Omega$ can be obtained.

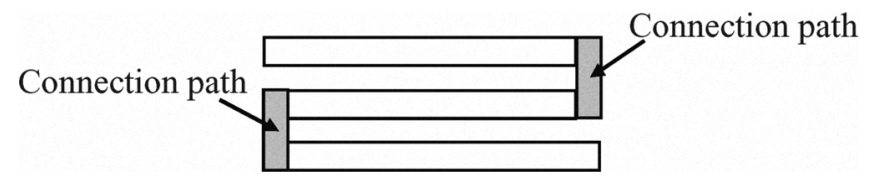

Figure 2. Sketch of the piezoresistor

Four piezoresistors are connected to construct a Wheatstone bridge in the way shown in Fig. 3 where the dashed lines indicate electrical connection and $\mathrm{P} 1 \sim \mathrm{P} 5$ indicate electrodes. $\mathrm{P} 5$ is the grounding, $\mathrm{P} 2$ is the input port, $\mathrm{P} 1$ and $\mathrm{P} 4$ are output ports, and an adjustable resistor will be connected between $\mathrm{P} 2$ and P3 to eliminate the unbalance of the bridge due to the nonuniform resistance of the piezoresistors, which is unavoidable in doping process.

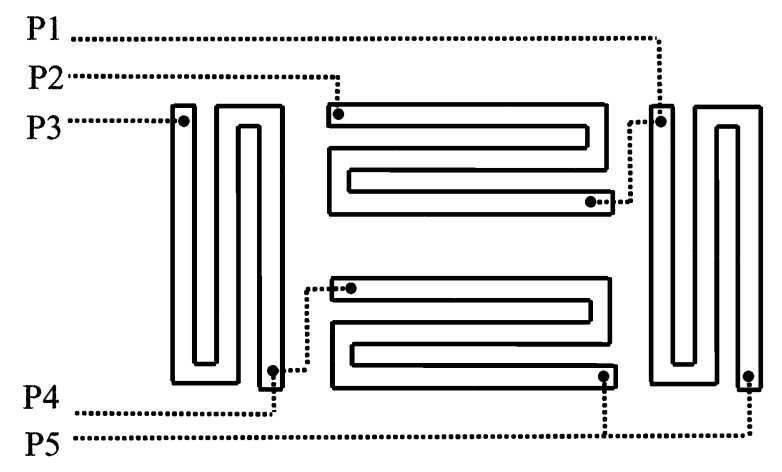

Figure 3. The construction of wheatstone bridge from the piezoersistors

\section{E. Simulation Results}

The configuration of the cantilever resonator is shown in Fig.4. Space is reserved to deposit gas sensing material later.

FEA simulation has been carried out to evaluate this configuration. The fundamental frequency obtained is about $14.2 \mathrm{kHz}$. Fig. 5 shows the FEA simulation result of the temperature distribution on the cantilever with $100 \mathrm{~mW}$ driving power. It can be seen that uniform temperature elevation is achieved in the gas sensing area. The stress produced at the root of the cantilever is about $3 \mathrm{MPa}$ which can provide sizable piezoresistive signal. 


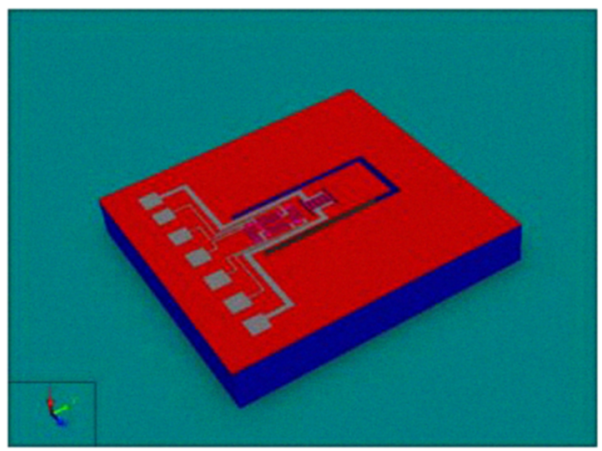

Figure 4. Skech of the cantilever resonator configuration
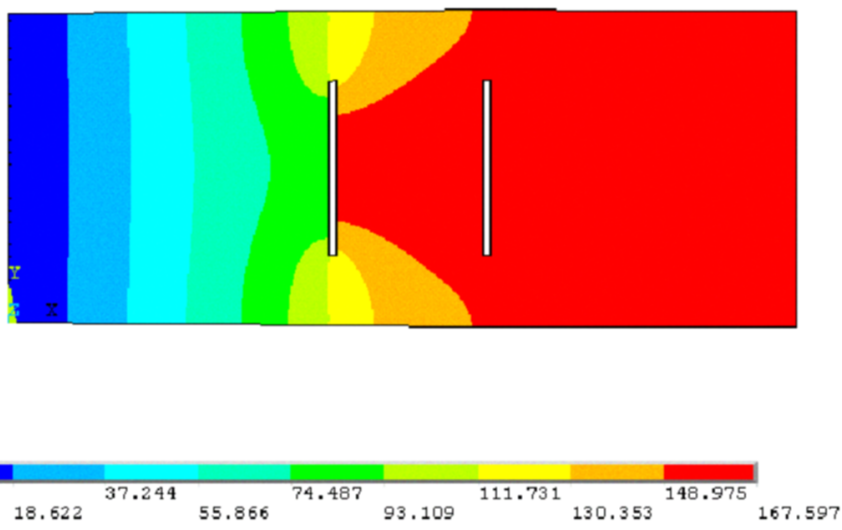

Figure 5. FEA simulation of temperature distribution on the cantilever with $100 \mathrm{~mW}$ driving power

\section{FABRICATION}

SOI wafer was used as the start material for the fabrication of the resonator. The advantages of SOI to MEMS are summarized in reference [8]. In this project, the SOI wafer is developed through directly bonding technique: an oxidated silicon wafer is bonded onto a non-oxidated silicon wafer and then grinded and polished to form a crystal-oxide-crystal sandwich structure, as show in Fig.6 (a). The thin crystal is as the front of the wafer and usually called device silicon, while the thick crystal is as the back of the wafer and called the substrate silicon.

The resistors, conduct paths and electrodes were fabricated on the device silicon. Boron implantation technology was used to produce resistors. A light doping process of $100 \Omega / \square$ sheet resistance and a heavy doping process of $10 \Omega / \square$ sheet resistance were used. Aluminium thin film was deposited by electron beam evaporation technology to make the conduct paths and electrodes. To obtain ideal doping distribution and real $\mathrm{Ohm}$ contact, anneal was performed in the processes.

The micromachining of the cantilever structure was accomplished by inductive coupled plasma (ICP) etching technology. The SOI wafer was etched from the front to form the cantilever dimension and subsequently from the back to provide the hanging height for the cantilever. The oxide between the device silicon and the substrate silicon acting as stop layer of silicon etching was etched in the end to release the cantilever structure. Actually the cantilever was developed through ICP etching successively the nitride film, the oxide film and the device silicon from the front of the wafer, and afterwards the substrate silicon and the oxide layer from the back of the wafer. The thickness of the cantilever was precisely guaranteed by the thickness of the device silicon.

The resistors, contacts, paths, pads and cantilever structure were patterned through lithography. The whole fabrication process included six lithographic steps defining the light doping areas, heavy doping areas, Ohm contacting areas, shape of electric paths and pads, front etching areas and back etching areas respectively. Therefore six lithographic masks $(\mathrm{M} 1 \sim \mathrm{M} 6)$ had to be developed. The fabrication processes of the resonator are schematicly presented in Fig. 6.

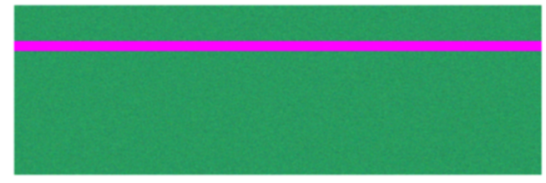

(a) The direct bonding SOI wafer

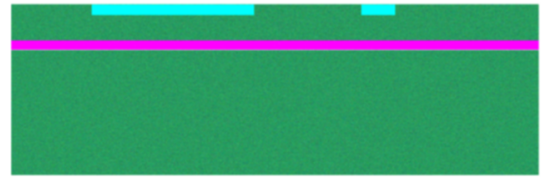

(b) Lightly doping Boron (M1)

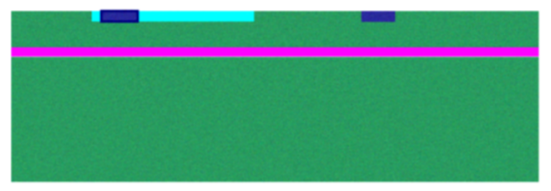

(c) Heavily doping Boron (M2)

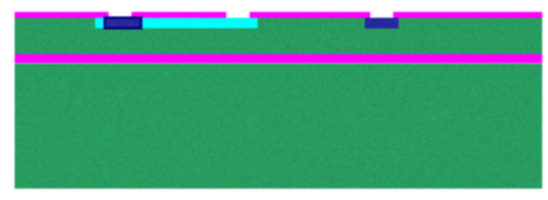

(d) Depositing and developing oxide film (M3)

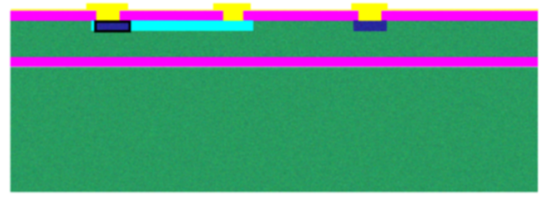

(e) Depositing and developing Al film (M4) 


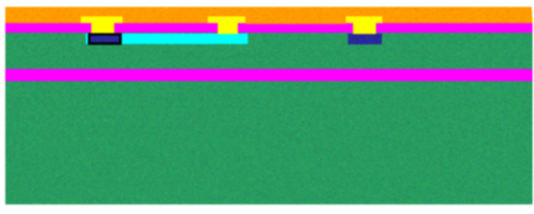

(f) Depositing nitride film

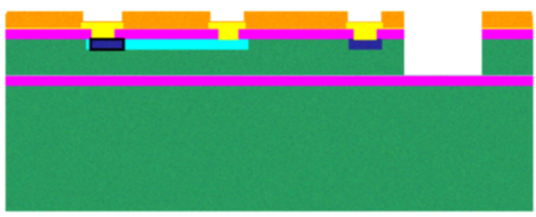

(g) ICP etching from the front (nitride, oxide, silicon) (M5)

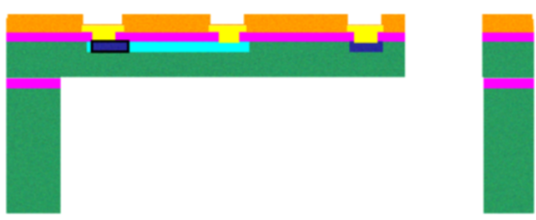

(h) ICP etching from the back (silicon, oxide) (M6)

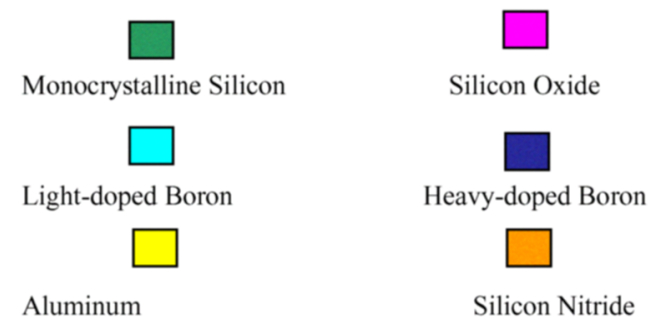

Figure 6. The schematilc fabrication processes of the resonator

Fig. 7 is the SEM picture of the resonator fabricated from the SOI wafer. The developed wafer was diced, sifted and package into chips. Fig. 8 is the picture of a resonator chip.

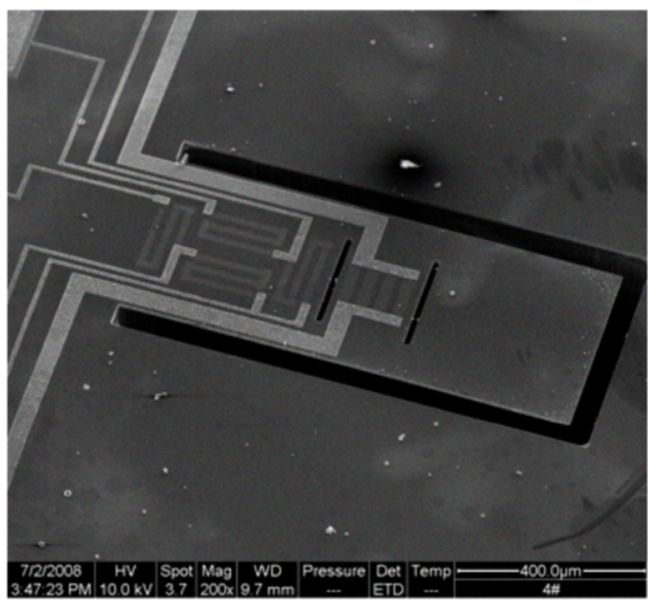

Figure 7. The SEM picture of the cantilever resonator

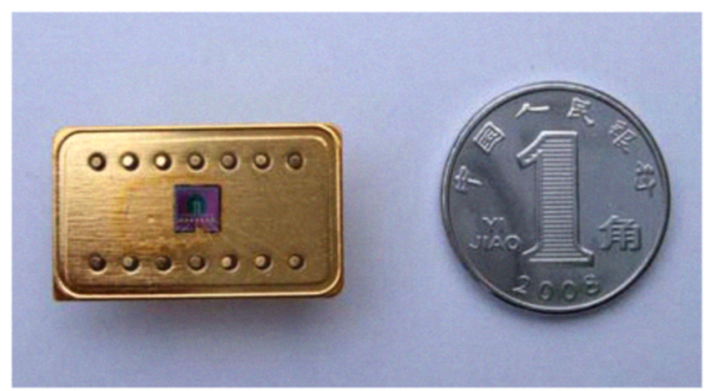

Figure 8. The packaged chip of the MEMS cantilever resonator

\section{TEST RESUlTS}

The amplitude-frequency characteristics of the cantilever resonator at different levels of excitation have been investigated. Fig.9 shows the amplitude-frequency characteristic of the resonator in ambient air under $3 \mathrm{~V}$ driving voltage. The resonant frequency measured is approximate $14.92 \mathrm{kHz}$ and the quality factor (Q) is approximate 200. According to the tested parameters of the resonator, nanogramlevel mass sensitivity can be expected, which provides the feasibility for trace gas detection. The cantilever resonator also shows advantages of high efficiency and stability in the test experiments.

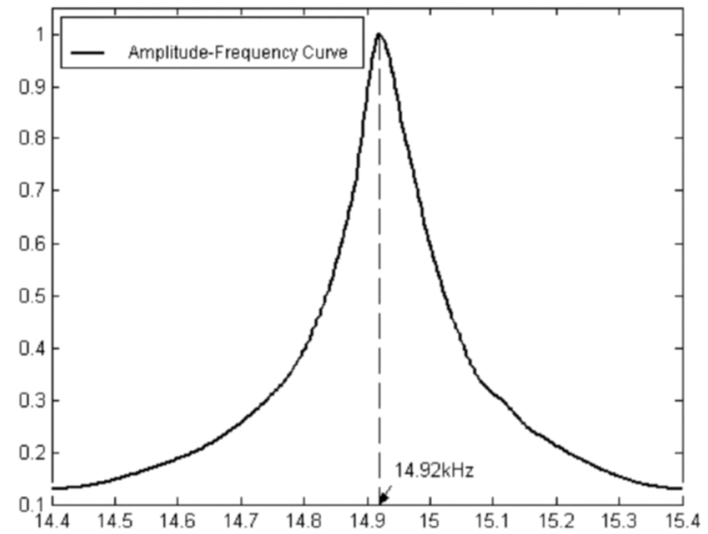

Figure 9. Tested amplitude-frequency characteristic of the resonator

\section{CONCLUSIONS}

The significant advantage of thermal driving and piezoresistive sensing mode over other operation modes of MEMS cantilever resonator is comparative testing signal can be produced with much lower driving voltage (below $5 \mathrm{~V}$ in this case), which permits practical application and provides opportunity of IC integration. The designed configuration for the MEMS cantilever resonator optimizes the sensitivity and the efficiency of the resonator and can create uniform 
temperature distribution for the function of the gas sensing material. SOI wafer provides a solution to the integrated fabrication of microstructure, transducers and electronics, and the precise control of the resonator parameters. The test results of the fabricated resonator agreed well with the theoretical calculation and FEA prediction. The resonator showed relatively high quality factor (approximate 200 in the air) in the experiment. According to the results, the proposed resonator is qualified to construct high sensitivity gas sensor.

With proper gas sensing material and close-loop vibration control circuit, the MEMS cantilever resonator will be made into specific trace gas sensors.

\section{ACKNOWLEDGMENT}

This project is supported by the National Nature Science Foundation of China (NSFC, No.50405060). The fabrication of the resonator was accomplished by the Institute of Semiconductors, Chinese Science Academy (CSA). We are grateful to Professor Ji An and Dr. Ning Jin for their advices and contributions.

\section{REFERENCES}

[1] N. V. Lavrik, "cantilever transducers as a platform for chemical and biological sensors," Review of Science Instruments, vol. 75, pp. 22292253, 2004.

[2] C. Ziegler, "cantilever-based biosensors," Anal. Bioanal. Chem., vol. 379, pp. 946-959, 2004.

[3] G. Stemme, "Resonant silicon sensors," J. Micromech. Microeng., vol. 1, pp. 113-125, 1991

[4] O. Brand and H. Baltes, "Micromachined resonant sensors- an overview," Sensors update, vol. 4, pp. 3-51, 1999

[5] H. P. Lang and C. Gerber, "Mechanical Sensors for the Nanoworld," Bionanotechnology Workshop and Meeting, Oxford University, UK, April 2002

[6] O. Brand, "CMOS-based resonant sensors," IEEE. sensors, Calffonda,USA, April 2005.

[7] Zhou Qin, "Study on MEMS Chemical Gas Sensor Based on Resonating Microcantilever", master's degree dissertation, Tsinghua University, 2006

[8] A.Y.Usenko, W.N.Carr, "Silicon-on-insulator technology for microelectromechanical applications", Semiconductor Physics, Quantum Electronics and Optoelectronics, vol.1, pp. 93-97, 1999 